Statistical Methods in Medical Research

\title{
A permutation test to analyse systematic bias and random measurement errors of medical devices via boosting location and scale models
}

2017, Vol. 26(3) 1443-1460

(C) The Author(s) 2015

Reprints and permissions: sagepub.co.uk/journalsPermissions.nav DOI: $10.1177 / 0962280215581855$ journals.sagepub.com/home/smm

\$SAGE

\author{
Andreas Mayr,' Matthias Schmid, ${ }^{2}$ Annette Pfahlberg,' \\ Wolfgang Uter' and Olaf Gefeller'
}

\begin{abstract}
Measurement errors of medico-technical devices can be separated into systematic bias and random error. We propose a new method to address both simultaneously via generalized additive models for location, scale and shape (GAMLSS) in combination with permutation tests. More precisely, we extend a recently proposed boosting algorithm for GAMLSS to provide a test procedure to analyse potential device effects on the measurements. We carried out a large-scale simulation study to provide empirical evidence that our method is able to identify possible sources of systematic bias as well as random error under different conditions. Finally, we apply our approach to compare measurements of skin pigmentation from two different devices in an epidemiological study.
\end{abstract}

\section{Keywords}

Measurement errors, systematic bias, random error, statistical models, permutation test, gradient boosting, regression

\section{Introduction}

When medical data or some characteristics are obtained using a medico-technical device, users expectation is typically that the measurements are precise and reflect the true status of what should be measured correctly. Measurement errors are, however, inevitable in practical situations. They are then subject of statistical analysis to learn about the measurement properties of the medicotechnical device. The analysis of measurements errors focuses on two different aspects, systematic bias and random error. ${ }^{1}$ Systematic bias is the component referring to a general difference between

\footnotetext{
'Institut für Medizininformatik, Biometrie und Epidemiologie, Friedrich-Alexander-Universität Erlangen-Nürnberg, Germany

${ }^{2}$ Institut für Medizinische Biometrie, Informatik und Epidemiologie, Rheinische Friedrich-Wilhelms-Universität Bonn, Germany

Corresponding author:

Andreas Mayr, Institut für Medizininformatik, Biometrie und Epidemiologie, Friedrich-Alexander-Universität Erlangen-Nürnberg, Waldstraße 6, 91054 Erlangen, Germany.

Email: andreas.mayr@fau.de
} 
the true value and the measurement in a specific direction. The occurrence of systematic bias hence has an impact on the validity of a measurement device. Random error, on the other hand, stands for the chance variability of the measurement. Random error may not lead to biased results as for large number of measurements it averages to zero; however, it is of concern when the reliability of a measurement device is considered.

A recent evaluation on measuring skin pigmentation, ${ }^{2}$ which was the motivation for our approach, compared two common devices (reflectometer RM100 and chromameter CR300) under various conditions. The authors compared mean values and coefficients of variation concluding that both devices were measuring basically the same (no difference in systematic bias) while one device had a lower variability (smaller random error).

We propose a new unified approach to simultaneously address both systematic bias and random error based on generalized additive models for location, scale and shape (GAMLSS). GAMLSS were introduced by Rigby and Stasinopoulos ${ }^{3}$ as a flexible semi-parametric approach to model not just the expected value ('location') of a conditional distribution (as in common generalized additive models $(\mathrm{GAM})^{4}$ ) but simultaneously up to four distribution parameters (also 'scale' and 'shape' parameters, hence the extension 'LSS'). The core of our approach is to model measurement data via two-dimensional location-scale GAMLSS to assess differences regarding systematic bias (device effect on location parameter) and random error (device effect on scale parameter) simultaneously.

While classical inference for GAMLSS is based on penalized maximum likelihood estimation, ${ }^{5}$ two alternative estimation techniques based on Bayesian inference ${ }^{6}$ or gradient boosting ${ }^{7}$ have been proposed recently. All three approaches may be used in this setting: the choice of the most appropriate one depends on the framework of the specific application.

GAMLSS have gained increasing interests over the last years: In a recent review article, ${ }^{8}$ GAMLSS were identified as one of the most powerful approaches beyond mean regression, which are more and more in the focus of methodological developments. ${ }^{9,6,10}$ In biomedical research, the most common application of GAMLSS is the construction of reference growth charts $^{11,12}$ for which it es even recommended by the World Health Organisation. ${ }^{13}$ Other recent applications include the analysis of genome-wide DNA methylation data ${ }^{14}$ or cognitive test data. $^{15}$

From a methodological point of view, a major issue in the context of GAMLSS is the simultaneous selection of the most influential predictors for the different distribution parameters. Variable selection is already a complicated issue in classical GAMs; in the case of GAMLSS this becomes even more pronounced due to the higher complexity of interdependent candidate models. The proposed gradient boosting algorithm ${ }^{7}$ particularly tackles this problem as it ensures simultaneous variable selection during the fitting process. Other advantages of boosting algorithms in general are their feasibility in high-dimensional settings with more predictors than observations $(p>n)$, and the implicit regularization leading to shrinkage of effect estimates. ${ }^{16,17}$ This increased flexibility, however, also leads to an important drawback: A limitation of the gradient boosting approach is the lack of standard errors for effect estimates (due to the shrinkage) which makes the construction of confidence intervals or significance tests problematic.

To overcome this problem, we extend the boosting algorithm by combining it with permutations of the grouping variable (i.e. the device) to construct a test procedure for the accuracy of measurements from different devices. To the best of the authors' knowledge, this is the first time that GAMLSS are considered in the context of analysing measurement errors as well as the first time that permutation tests are combined with multidimensional gradient boosting. 
This article is structured as follows. First, we provide the methodological background of our approach by shortly describing the model class and the underlying boosting algorithm with the permutation procedure. In a second step, we present results of a large scale simulation study (including high-dimensional settings) to provide evidence that our approach is able to correctly identify possible sources of systematic bias and random error under different conditions. Finally, we illustrate the new testing procedure in an epidemiological study in order to identify differences between two devices to measure skin pigmentation. ${ }^{2}$

\section{Methods}

\section{I Assessing the accuracy of measurements via GAMLSS}

Analysing the accuracy of measurements from medico-technical devices is an important task in medical research. The two most used terms in this context are validity and reliability. While the validity of a device refers to its ability to actually measure the parameter of interest, reliability of a device describes its stability. Generally, the total measurement error can be decomposed into systematic bias and random error, i.e.

$$
\text { measurement error }=\text { systematic bias }+ \text { random error }
$$

Systematic bias refers to a general trend of the device to overestimate or underestimate the true parameter of interest. The common procedure to assess this bias is to compare measurements $\tilde{y}_{1}^{A}, \ldots, \tilde{y}_{n}^{A}$ for parameter $Y$ from the device $A$ with true values $y_{1}, \ldots, y_{n}$ where $n$ denotes the sample size. If the main goal is limited to identify the bias of the device, these comparisons are often based on comparing mean values in combination with univariate statistical tests. For a more profound analysis, taking possible subject-specific covariates or confounders $X=x_{1}, \ldots, x_{p}$ into account, one could consider regressing the measurements and true values to these covariates while considering the device as grouping variable:

$$
\mu=E(Y \mid X, \text { device })=\beta_{0}+\sum_{j=1}^{p} h_{j}\left(x_{j}\right)+\beta_{s b}(\operatorname{device}=A)
$$

Here, $h_{j}(\cdot)$ denotes the effect of covariate $x_{j}$ on the outcome $Y, I(\cdot)$ is an indicator function and $\beta_{\mathrm{sb}}$ represents an estimate for the possible systematic bias of device $A$. If the measurements or true values are from the same patients, additional subject-specific 'random' effects might be necessary to adjust for repeated measurements.

The assessment of random error, which refers to the variability of the measurement, can be tackled by analysing repeated measurements of one device (yielding an estimate of the reliability of this device) or by comparing the variation of measurements from different devices (possibly aiming at identifying the most reliable device).

We propose an alternative method to address both systematic bias and random error simultaneously in a unified regression framework. The concept is based on GAMLSS. GAMLSS consider separate additive models for all parameters of the conditional distribution, including scale and shape parameters, thereby extending classical regression which only focuses on the mean $\mu$.

When evaluating the properties of measurement data, we want to take advantage of this increased flexibility, by extending the equation (1) for systematic bias to assess also random error. 
Consider again measurements for parameter $Y$ and $Y \sim N\left(\mu, \sigma^{2}\right)$, then we can model not only an effect of the device on the expected value $\mu$ but simultaneously also, via a log-link, on the standard deviation $\sigma$ :

$$
\begin{array}{r}
\mu=E(Y \mid X, \text { device })=\beta_{0 \mu}+\sum_{j=1}^{p} h_{\mu j}\left(x_{j}\right)+\beta_{\mathrm{sb}}(\text { device }=A) \\
\log (\sigma)=\log (\sqrt{\operatorname{Var}(Y \mid X, \text { device })})=\beta_{0 \sigma}+\sum_{j=1}^{p} h_{\sigma j}\left(x_{j}\right)+\beta_{\text {re }}(\text { device }=A)
\end{array}
$$

The coefficient $\beta_{\mathrm{sb}}$ hence represents an estimate for the systematic bias of the device while $\beta_{\mathrm{re}}$ represents the effect the device contributes to the random error of the measurement.

An important special case in the analysis of measurement errors, that can be often found in practice, is the comparison of measurements from different devices: When no true values are available, one can model the measurements $\left(\tilde{y}_{1}^{A}, \tilde{y}_{1}^{B}\right), \ldots,\left(\tilde{y}_{n}^{A}, \tilde{y}_{n}^{B}\right)$ from different devices with the same approach presented above. However, the interpretation of the resulting coefficient $\beta_{\mathrm{sb}}$ changes. An effect of device $\mathrm{A}$ on $\mu$ in this case represents a systematic difference between the two devices, leading to the conclusion that at least one of the devices incorporates a systematic bias. Without true values, it is, however, not possible to tell which of the devices leads to smaller bias. In the case of $\beta_{\text {re }}$ the model without true values is able to identify the device which leads to a smaller random error.

Note that the basic idea of this approach is not limited to normally distributed measurements but can be adapted to other distributions in the GAMLSS framework. Examples could be the beta distribution for bounded continuous measurements (like proportions) or the negative binomial distribution for count data. ${ }^{18,19}$ The most obvious cases are distributions where single parameters can be directly linked to either location or scale, here the interpretation is identical to the presented Gaussian setting. For distributions where multiple parameters contribute to the expected value or the variance, respectively, a device effect on any of these parameters represents systematic bias or random error, respectively. In case of a skewness parameter that contributes to both, location and the scale, a device effect on this parameter can be interpreted as a possible source of both, systematic bias and random error.

\subsection{Boosting beyond the mean}

For inference, the developers of the GAMLSS model class provided two penalized maximum likelihood algorithms based on backfitting, ${ }^{20}$ incorporating also automatic smoothing parameter selection for nonlinear effects. ${ }^{5}$ One of the main drawbacks of these classical algorithms is that they can not cope with high-dimensional data. Step-wise variable selection techniques based on information criteria are available, however, they are limited to moderate numbers of candidate variables. Note that variable selection for GAMLSS is a far more complex task than in classical GAMs, as variables for up to four different additive predictors have to be selected simultaneously.

To overcome this problem, a recent article $^{7}$ proposed a boosting algorithm that can incorporate variable selection and is feasible for high-dimensional data. The concept of boosting emerged from machine learning and was later adapted to estimate the unknown quantities in statistical models. ${ }^{21}$ Over the last few years, these statistical boosting algorithms have been further extended to various regression settings and were specifically adapted to medical research questions. ${ }^{22}$

The main idea is to iteratively apply simple regression tools (denoted as base-learners) for each candidate variable. In each iteration, only the best-performing base-learner (and hence only the most 
informative predictor) is selected and a small proportion of its fit is included in the current model. By doing so, the algorithm additionally incorporates shrinkage of effect estimates, which typically leads to a reduced variance of estimates and should therefore increase the stability and accuracy of predictions. ${ }^{23}$ The main tuning parameter of statistical boosting algorithms is their stopping

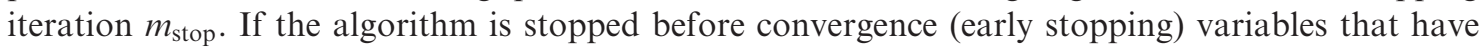
never been included in the model up to this point are excluded. As the base-learners can be selected various times, also the amount of shrinkage and even the smoothness of spline-based base-learners depends on the selection of $m_{\text {stop }}$. The selection of $m_{\text {stop }}$ hence reflects the common bias-variance trade-off in statistical modelling: large values of $m_{\text {stop }}$ lead to more complex models with higher variance and small bias; smaller values of $m_{\text {stop }}$ lead to sparser models with fewer selected variables, more shrinkage and reduced variance. ${ }^{24}$

The adapted boosting algorithm to fit GAMLSS circles through the different distribution parameters, carrying out one boosting iteration for each parameter dimension. In this case it can be advantageous to select different stopping iterations for the different parameter dimensions by searching a pre-specified grid of possible combinations. ${ }^{7}$ The complete algorithm for boosting GAMLSS can be found in the appendix, the implementation is available in the $\mathrm{R}^{25}$ add-on package gamboostLSS. $^{26}$

Although statistical boosting can be considered as one of the most flexible inference schemes in the toolbox of a modern statistician, ${ }^{21}$ this increased flexibility comes with an important drawback: due to shrinkage and variable selection implied by early stopping, it is extremely difficult to derive estimates for the standard deviation of predictor effects. ${ }^{7}$ As a result, currently, statistical boosting cannot be applied in settings where it is necessary to assess the significance of single predictor effects such as $\beta_{\mathrm{sb}}$ and $\beta_{\text {re. }}$.

\subsection{Permutation test}

To overcome the problem of inference for boosting GAMLSS, we propose a simple permutation algorithm to simultaneously derive $p$-values to assess device effects both on the location and the scale parameters. Permutation tests in regression settings have a long history in statistical literature. ${ }^{27}$ Often they were proposed for small sample sizes where traditional asymptotic tests become problematic. An overview on different methodological aspects of permutation tests was provided by Kennedy and Cade. ${ }^{28}$

In order to test the hypothesis that a regression coefficient $\beta_{j}$ for a covariate $z_{j}$ is zero $\left(H_{0}: \beta_{j}=0\right.$ vs. $\left.H_{1}: \beta_{j} \neq 0\right)$, the basic idea is to generate an empirical distribution of the test statistic under the null hypothesis via permutations. This distribution is then applied to estimate the probability that the test statistic representing the effect of $z_{j}$ under the null is larger or equal the observed effect ( $p$-value).

Permutation tests for regression settings mainly differ regarding two aspects: (i) what is exactly permutated; (ii) what test statistic is used.

Regarding aspect (i), three different possibilities exist. The first two are to permute either the response $Y$ or the covariate $z_{j}$. If the underlying model is univariate, both approaches approximately lead to the same results. If the model contains various correlated predictors, a third possibility is to replace $z_{j}$ by the residuals $r_{j}$ of $z_{j}$ given all other predictors. These residuals are then permutated, generating a distribution under the null hypothesis while also destroying possible correlations (as $r_{j}$ is an orthogonal projection on $z_{j}$ ). ${ }^{29,30}$ In the special case of assessing measurement errors, the indicator variable for the different devices $I($ device $=A)$ is by all means uncorrelated to the other covariates. The other predictors may have an effect on the accuracy of the measurement but not on 
the selection of the device. Due to this independence, applying permutation of residuals $r_{j}$ or the actual covariate $z_{j}$ (i.e. the indicator variable) leads to the same results. Our method will be therefore based on simply permutating the indicator variable (i.e. $z_{j}$ ).

Regarding aspect (ii), many authors propose to incorporate asymptotically pivotal test statistics such as the $t$-statistic or the likelihood ratio, others simply use the estimated coefficient $\hat{\beta}_{j}{ }^{28}$ In the case of a two-dimensional GAMLSS, where $z_{j}$ contributes both via location and scale parameter to the likelihood (e.g. $\mu$ and $\sigma$ ) it makes sense to avoid the likelihood ratio in order to get different $p$-values for the different additive predictors instead of an overall effect of $z_{j}$. The $t$-statistic,

$$
t=\frac{\left|\hat{\beta}_{j}\right|}{\sqrt{\operatorname{Var}\left(\hat{\beta}_{j}\right)}}
$$

on the other hand, is concordant to $\hat{\beta}_{j}$ in univariate settings or if $z_{j}$ is independent from the other predictors. In these cases, the resulting $p$-values should hence be exactly the same. In our case, where we assume the choice of the device being independent from the other predictors, we therefore stick to simply using the estimated coefficients $\hat{\beta}_{\mathrm{sb}}$ and $\hat{\beta}_{\text {re }}$ as test statistics. The proposed permutation test hence works as follows.

Step 1: Fit the original GAMLSS via boosting:

$$
\begin{gathered}
\hat{\mu}=\hat{\beta}_{0 \mu}+\sum_{j=1}^{p} \hat{h}_{\mu j}\left(x_{j}\right)+\hat{\beta}_{\mathrm{sb}} \cdot I(\text { device }=A) \\
\log (\hat{\sigma})=\hat{\beta}_{0 \sigma}+\sum_{j=1}^{p} \hat{h}_{\sigma j}\left(x_{j}\right)+\hat{\beta}_{\mathrm{re}} \cdot I(\text { device }=A)
\end{gathered}
$$

Step 2: Permute the device indicator $B$ times. Apply boosting to estimate the GAMLSS with the permutated indicator variables, leading to

$$
\hat{\beta}_{\mathrm{sb}}^{(1)}, \ldots, \hat{\beta}_{\mathrm{sb}}^{(B)} \text { and } \hat{\beta}_{\mathrm{re}}^{(1)}, \ldots, \hat{\beta}_{\mathrm{re}}^{(B)}
$$

If the corresponding variables were not selected by the boosting algorithm, the coefficients are set to zero.

Step 3: Compute $p$-values to assess a device effect on $\mu$ and $\sigma$ :

$$
\begin{aligned}
& p \text {-value }(\mathrm{sb})=\frac{1}{B} \sum_{b=1}^{B} I\left(\left|\hat{\beta}_{\mathrm{sb}}^{(b)}\right| \geq\left|\hat{\beta}_{\mathrm{sb}}\right|\right) \\
& p \text {-value }(\mathrm{re})=\frac{1}{B} \sum_{b=1}^{B} I\left(\left|\hat{\beta}_{\mathrm{re}}^{(b)}\right| \geq\left|\hat{\beta}_{\mathrm{re}}\right|\right)
\end{aligned}
$$


Note that both the original coefficients $\left(\hat{\beta}_{\mathrm{sb}}, \hat{\beta}_{\mathrm{se}}\right)$ and those from the permutations $\left(\hat{\beta}_{\mathrm{sb}}^{(b)}, \hat{\beta}_{\mathrm{re}}^{(b)}, b=1, \ldots, B\right)$ will be affected by shrinkage. By construction, the permutation test is only based on the rankings of the coefficients. If all coefficients are shrunk in the same manner (same stopping iteration $m_{\text {stop }}$ ) this should not influence the performance of the permutation test. We will investigate this issue in the next section.

\section{Simulation study}

We first carried out a simulation study to investigate the performance of our approach under controlled conditions where the true results are known. The main aims of the simulation study were:

(i) to investigate whether the proposed approach is able to detect possible device effects on the expected values (systematic bias) and the variance (random error) of measurements;

(ii) to check whether the permutation test can hold the type I error rate under different conditions, including also high-dimensional data situations;

(iii) to investigate the effect of the stopping iterations $m_{\text {stop }}$ and the resulting shrinkage on the performance of the algorithm.

\section{I Settings}

The response observations were simulated from a normal distribution $Y \sim N\left(\mu, \sigma^{2}\right)$. Both the expected value $\mu$ and the variance $\sigma^{2}$ were affected by a continuous uniformly distributed covariate $X \sim U(0,1)$. In every simulation step we generated independent samples with different sample sizes $n=\{50,200,500\}$. Always half of the sample is considered as true values $y_{i}, \ldots, y_{n}$, while the other half represents measurements from a device $\tilde{y}_{\frac{n}{2}+1}, \ldots, \tilde{y}_{n}$. The variable $d_{i} \in\{0,1\}$ is the device indicator.

We considered four different simulation settings to investigate the questions presented above. An overview of the resulting distributions of the true and values and measurements from the device can be found in Figure 1.

\section{Setting 1:}

No device effect, neither on expected value nor on variance:

$$
\mu_{i}=2+3 x_{i}, \quad \sigma_{i}=\exp \left(0.5 x_{i}\right)
$$

\section{Setting 2:}

Device effect leading to systematic bias:

$$
\mu_{i}=2+3 x_{i}-0.5 d_{i}, \quad \sigma_{i}=\exp \left(0.5 x_{i}\right)
$$

\section{Setting 3:}

Device effect leading to higher random error:

$$
\mu_{i}=2+3 x_{i}, \quad \sigma_{i}=\exp \left(0.5 x_{i}+0.5 d_{i}\right)
$$




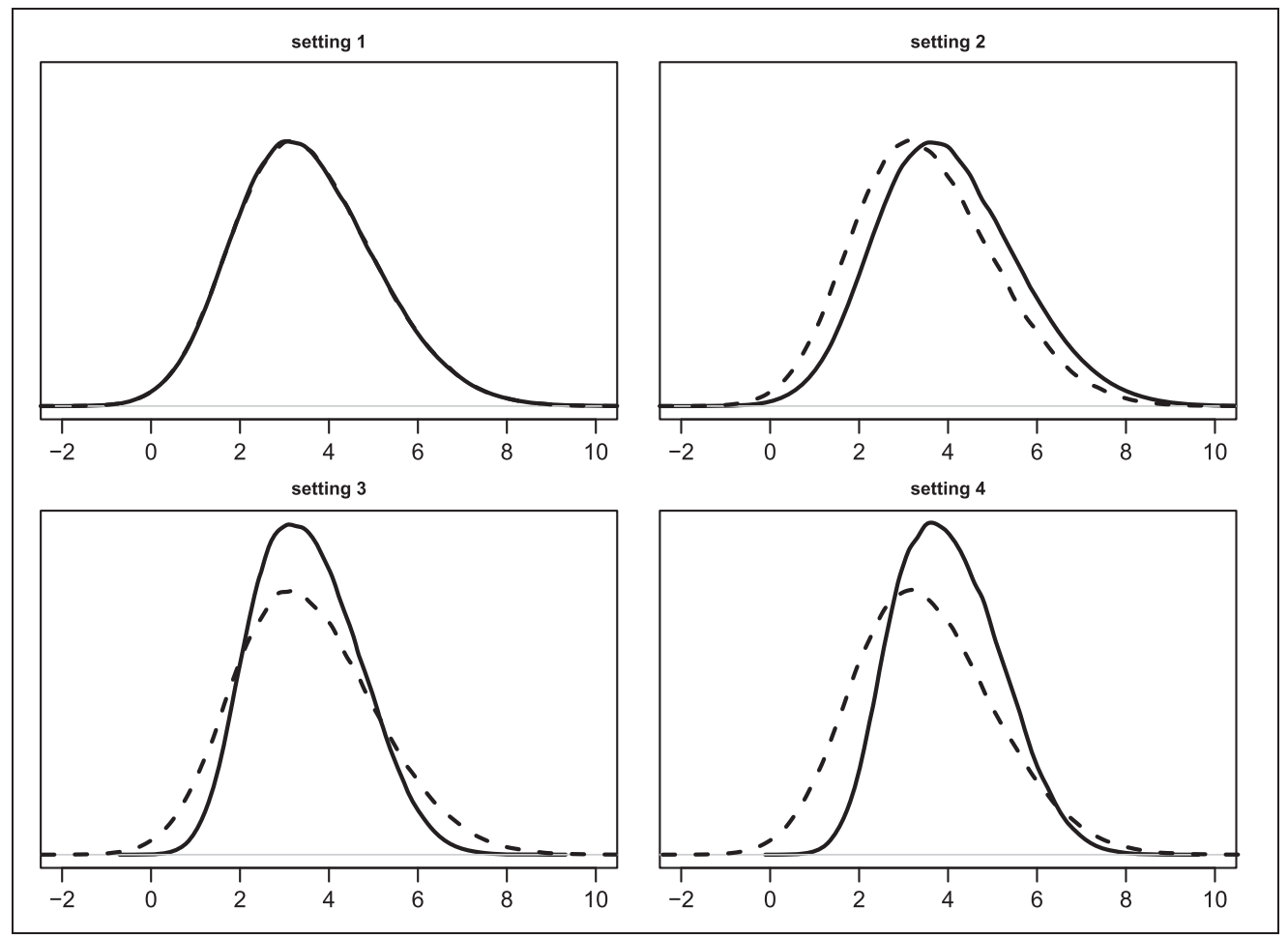

Figure I. Overview on the four different simulation settings. Curves represent the distributions of the true values (solid lines -) and measurements from a device (dashed lines - -).

\section{Setting 4:}

Device effect leading both to systematic bias and higher random error:

$$
\mu_{i}=2+3 x_{i}-0.5 d_{i}, \quad \sigma_{i}=\exp \left(0.5 x_{i}+0.5 d_{i}\right)
$$

For each setting, we generated 1000 different samples of the outcome distributions and fitted on each a Gaussian location-scale GAMLSS via boosting. The covariate $x$ and the device indicator $d_{i}$ were included in the additive predictors for both $\mu$ and $\sigma$ via separate ordinary least-squares baselearners. The best combination of stopping iterations $m_{\text {stop }}$ was then either selected from a twodimensional logarithmic grid via bootstrapping or $\left(m_{\text {stop }}=\mathrm{CV}\right)$ algorithm was stopped after a pre-specified number of iterations $m_{\text {stop }}=\{100,500,1000\}$.

Afterwards, the proposed permutation test was performed by permutating the device indicator $B=1000$ times and fitting the same model again with the same number of boosting iterations. The resulting 1000 new coefficients $\hat{\beta}_{\mathrm{sb}}^{(1)}, \ldots, \hat{\beta}_{\mathrm{sb}}^{(B)}$ and $\hat{\beta}_{\mathrm{re}}^{(1)}, \ldots, \hat{\beta}_{\mathrm{re}}^{(B)}$ from the base-learner representing the device indicator were then simultaneously compared with the coefficient from the original model in order to compute the $p$-values. The simulation design hence results in separate $p$-values indicating possible effects of the device on systematic bias $(\mu)$ and random error $(\sigma)$ for each of the 1000 simulation runs.

To put the results of our approach into perspective, we additionally fitted the same models with the classical penalized maximum likelihood approach (penalized ML) implemented in the R add-on 
Table I. Results from the simulation study: $p$-values. Median $p$-values from the classical parametric test of the penalized maximum likelihood (ML) approach and the new permutation test for the boosting approach. Results refer to the four different simulation settings (set.), different sample sizes $(n)$, different stopping iterations $\left(m_{\text {stop }}\right)$ and different numbers of predictors $(p)$.

\begin{tabular}{|c|c|c|c|c|c|c|c|c|c|c|c|c|}
\hline \multirow[b]{2}{*}{ set. } & \multirow[b]{2}{*}{$n$} & \multirow[b]{2}{*}{$p$} & \multicolumn{2}{|c|}{ penalized ML } & \multicolumn{2}{|c|}{$m_{\text {stop }}=100$} & \multicolumn{2}{|c|}{$m_{\text {stop }}=500$} & \multicolumn{2}{|c|}{$m_{\text {stop }}=1000$} & \multicolumn{2}{|c|}{$m_{\text {stop }}=C V$} \\
\hline & & & $\mathrm{sb}(\mu)$ & $\operatorname{re}(\sigma)$ & $\operatorname{sb}(\mu)$ & $\operatorname{re}(\sigma)$ & $\operatorname{sb}(\mu)$ & $\operatorname{re}(\sigma)$ & $\mathrm{sb}(\mu)$ & $\operatorname{re}(\sigma)$ & $\operatorname{sb}(\mu)$ & $\operatorname{re}(\sigma)$ \\
\hline I & 50 & 2 & 0.510 & 0.484 & 0.526 & 0.514 & 0.532 & 0.500 & 0.532 & 0.500 & 0.586 & 0.570 \\
\hline 2 & 50 & 2 & 0.162 & 0.484 & 0.175 & 0.515 & 0.182 & 0.504 & 0.182 & 0.502 & 0.172 & 0.540 \\
\hline 3 & 50 & 2 & 0.508 & 0.020 & 0.533 & 0.033 & 0.534 & 0.035 & 0.534 & 0.035 & 0.517 & 0.088 \\
\hline 4 & 50 & 2 & 0.093 & 0.020 & 0.102 & 0.032 & 0.110 & 0.034 & 0.110 & 0.034 & 0.105 & 0.088 \\
\hline I & 200 & 2 & 0.483 & 0.487 & 0.488 & 0.484 & 0.486 & 0.482 & 0.486 & 0.482 & 0.512 & 0.570 \\
\hline 2 & 200 & 2 & 0.006 & 0.487 & 0.006 & 0.488 & 0.006 & 0.478 & 0.006 & 0.478 & 0.006 & 0.540 \\
\hline 3 & 200 & 2 & 0.487 & 0.000 & 0.488 & 0.000 & 0.488 & 0.000 & 0.488 & 0.000 & 0.491 & 0.000 \\
\hline 4 & 200 & 2 & 0.001 & 0.000 & 0.001 & 0.000 & 0.001 & 0.000 & 0.001 & 0.000 & 0.001 & 0.000 \\
\hline I & 500 & 2 & 0.525 & 0.518 & 0.524 & 0.522 & 0.523 & 0.524 & 0.523 & 0.524 & 0.528 & 0.525 \\
\hline 2 & 500 & 2 & 0.000 & 0.518 & 0.000 & 0.528 & 0.000 & 0.522 & 0.000 & 0.522 & 0.000 & 0.527 \\
\hline 3 & 500 & 2 & 0.524 & 0.000 & 0.522 & 0.000 & 0.526 & 0.000 & 0.526 & 0.000 & 0.531 & 0.000 \\
\hline 4 & 500 & 2 & 0.000 & 0.000 & 0.000 & 0.000 & 0.000 & 0.000 & 0.000 & 0.000 & 0.000 & 0.000 \\
\hline I & 200 & 200 & - & - & I & I & I & I & 0.526 & 0.488 & 1 & 1 \\
\hline 2 & 200 & 200 & - & - & 0.010 & I & 0.022 & I & 0.025 & 0.500 & 0.008 & 1 \\
\hline 3 & 200 & 200 & - & - & I & 0.000 & 0.446 & 0.000 & 0.465 & 0.000 & I & 0.000 \\
\hline 4 & 200 & 200 & - & - & 0.001 & 0.000 & 0.002 & 0.000 & 0.003 & 0.000 & 0.001 & 0.000 \\
\hline
\end{tabular}

package gamlss ${ }^{20}$ and compared the $p$-values resulting from the permutation tests to the corresponding parametric Wald-type tests implemented there.

In order to investigate the performance of the permutation test in case of high-dimensional data, we repeated all four settings with $n=200$ (100 true values and measurements, respectively) and $p=200$ predictors. We therefore additionally included also 198 non-informative pairwise correlated $(\rho=0.5)$ normally distributed covariates, which did not have an effect neither on $\mu$ nor on $\sigma$. For these data settings the comparison with the likelihood-based approach, however, was impossible as high-dimensional GAMLSS currently can only be fitted via boosting: note that in the context of location and scale models, a dimensionality of $p=200$ means that the algorithm has to estimate more than 400 unknown quantities.

\subsection{Results}

The resulting $p$-values from the new approach together with the corresponding parametric procedure from the likelihood-based fitting are presented in Table 1. At first glance, it appears that although the methodological background of the parametric tests and the permutation tests following gradient boosting are completely different, the resulting median $p$-values are more or less identical, especially for the larger sample sizes $n=200$ and $n=500$. For $n=50$ (25 true values and 25 measurements) the differences between the different procedures are more pronounced.

Concerning the question of whether the new approach is truly able to detect effects of a measuring device on systematic bias and random error the answer again depends on the sample size. For the larger sample sizes the resulting median $p$-values in case of true effects (systematic bias, settings 2 
Table 2. Results from the simulation study: type I error rate. Mean proportion of simulation iterations where the test procedure yielded a $p$-value smaller than $\alpha=0.05$, although there did not exist a true device effect (otherwise $\cdot$ ).

\begin{tabular}{|c|c|c|c|c|c|c|c|c|c|c|c|c|}
\hline \multirow[b]{2}{*}{ set. } & \multirow[b]{2}{*}{$n$} & \multirow[b]{2}{*}{$p$} & \multicolumn{2}{|c|}{ penalized ML } & \multicolumn{2}{|c|}{$m_{\text {stop }}=100$} & \multicolumn{2}{|c|}{$m_{\text {stop }}=500$} & \multicolumn{2}{|c|}{$m_{\text {stop }}=1000$} & \multicolumn{2}{|c|}{$m_{\text {stop }}=C V$} \\
\hline & & & $\mathrm{sb}(\mu)$ & $\operatorname{re}(\sigma)$ & $\mathrm{sb}(\mu)$ & $\operatorname{re}(\sigma)$ & $\operatorname{sb}(\mu)$ & $\operatorname{re}(\sigma)$ & $\mathrm{sb}(\mu)$ & $\operatorname{re}(\sigma)$ & $\mathrm{sb}(\mu)$ & $\operatorname{re}(\sigma)$ \\
\hline I & 50 & 2 & 0.074 & 0.061 & 0.053 & 0.050 & 0.057 & 0.051 & 0.057 & 0.051 & 0.062 & 0.048 \\
\hline 2 & 50 & 2 & . & 0.061 & . & 0.053 & . & 0.055 & . & 0.055 & . & 0.044 \\
\hline 3 & 50 & 2 & 0.068 & . & 0.057 & . & 0.059 & . & 0.059 & & 0.059 & . \\
\hline I & 200 & 2 & 0.052 & 0.055 & 0.048 & 0.057 & 0.053 & 0.055 & 0.053 & 0.055 & 0.048 & 0.062 \\
\hline 2 & 200 & 2 & . & 0.055 & . & 0.055 & . & 0.053 & . & 0.053 & . & 0.060 \\
\hline 3 & 200 & 2 & 0.051 & $\cdot$ & 0.052 & . & 0.051 & . & 0.051 & 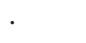 & 0.046 & . \\
\hline I & 500 & 2 & 0.039 & 0.049 & 0.040 & 0.050 & 0.038 & 0.050 & 0.038 & 0.050 & $0.04 I$ & 0.045 \\
\hline 2 & 500 & 2 & & 0.049 & . & 0.045 & 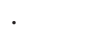 & 0.049 & . & 0.049 & . & 0.049 \\
\hline 3 & 500 & 2 & 0.045 & $\cdot$ & 0.044 & . & 0.044 & $\cdot$ & 0.044 & $\cdot$ & 0.043 & . \\
\hline I & 200 & 200 & - & - & 0.048 & 0.057 & 0.046 & 0.045 & 0.050 & 0.047 & 0.044 & 0.047 \\
\hline 2 & 200 & 200 & - & - & . & 0.055 & . & 0.044 & . & 0.042 & . & 0.045 \\
\hline 3 & 200 & 200 & - & - & 0.077 & . & 0.061 & . & 0.061 & & 0.076 & . \\
\hline
\end{tabular}

and 4; random error, settings 3 and 4 ) are all close to 0 . This holds also true for the high-dimensional data situations with $p=200$. Only for the smaller sample size $(n=50)$ the approach seems to have problems to detect the effects on systematic bias. The median $p$-values for the effects on random error (effects on $\sigma$ ) on the other hand is still below 0.05. The same difficulties, however, can be also observed for the parametric procedure.

These results are further supported by the estimated type I error rates: The proportion of simulation steps where the resulting $p$-values fell below $\alpha=0.05$ although there was no true device effect (settings 1-3) are presented in Table 2. One can again clearly observe the effect of the sample size-however, this time only for the parametric test. For the larger sample sizes $n=200$ and $n=500$ both the new approach as well as the parametric tests seem to hold the type I error rate. For smaller sample sizes $(n=50)$ the type I error rates of the parametric test procedure clearly is above $\alpha=0.05$. The boosting procedure with permutation test, on the other hand, seems to be able to hold the $\alpha$-level also for smaller sample sizes. Even for $n=50$ the empirical distribution of the resulting $\mathrm{p}$-values from setting 1 , where there is no true effect neither on systematic bias or random error, follow a univariate $U(0,1)$ distribution (results not shown).

Regarding the selection of the stopping iteration $m_{\text {stop }}$, the results in both tables suggest that for low-dimensional data situations there is only a minor effect on the resulting $p$-values. The more timeconsuming variant where we used the combination of stopping iterations which optimized the prediction error of the initial model in a bootstrap analysis $\left(m_{\text {stop }}=\mathrm{CV}\right)$ sometimes even yielded slightly poorer results than just using fixed values of $m_{\text {stop }}$ (e.g. setting 1 , Table 2 ).

Overall, the performance of our approach is rather robust with respect to the selection of the

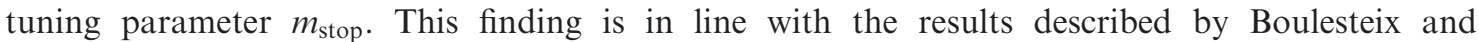
Hothorn. ${ }^{31}$ Although the size of the coefficients is affected by shrinkage and therefore depends on $m_{\text {stop }}$, the results of the permutation tests are mainly based on the rankings of the coefficients $\hat{\beta}_{\mathrm{sb}}^{(1)}, \ldots, \hat{\beta}_{\mathrm{sb}}^{(B)}$ and $\hat{\beta}_{\mathrm{re}}^{(1)}, \ldots, \hat{\beta}_{\mathrm{re}}^{(B)}$ compared to the initial estimates $\hat{\beta}_{\mathrm{sb}}$ and $\hat{\beta}_{\mathrm{re}}$. This ranking, however, does not change much if the same stopping iterations is used for the initial model and the permutations (see Figure 2). 


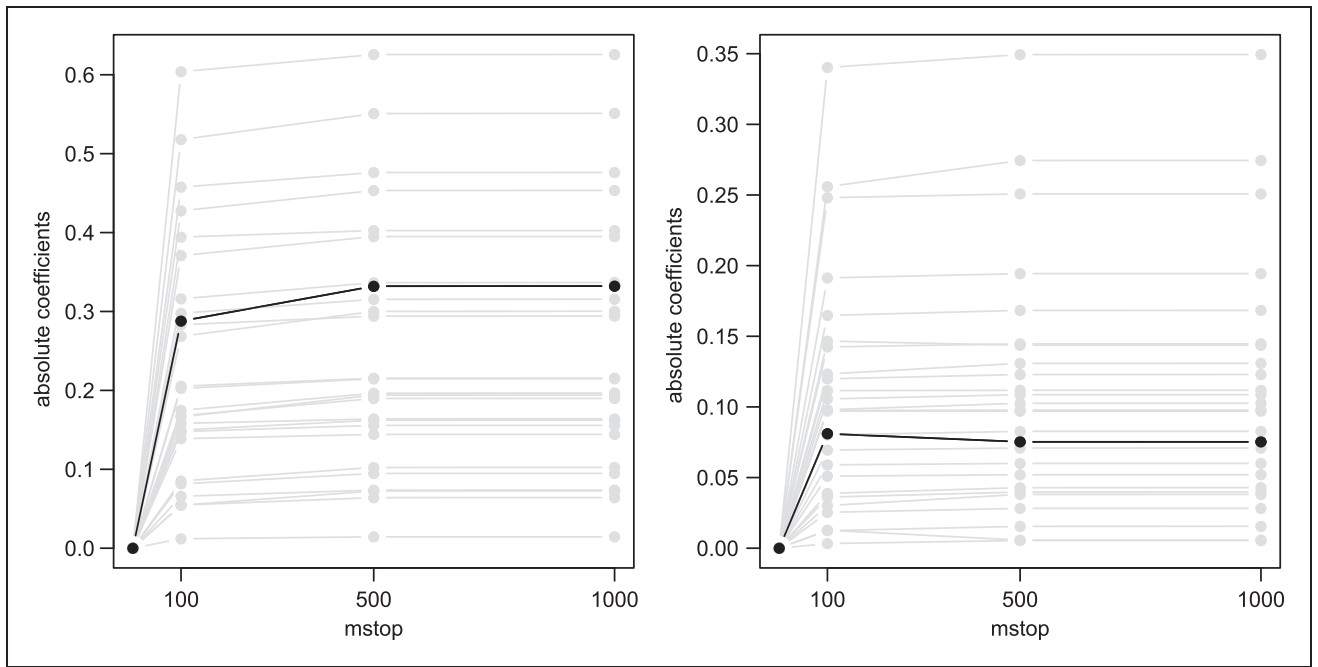

Figure 2. Effect of the stopping iteration $m_{\text {stop. }}$. Points refer to the absolute coefficients $\left|\hat{\beta}_{\text {sb }}\right|$ (left) and $\left|\hat{\beta}_{\text {re }}\right|$ (right) from the original model (dark lines) and 25 permutations (grey lines) from the simulation setting I (no device effect, neither on systematic bias or random error) with $n=50$.

In the case of high-dimensional data $(p=200)$, in many simulation steps the non-informative device variable was not selected at all for $m_{\text {stop }}=100$ or $m_{\text {stop }}=500$. This inevitably leads to a $p$-value of 1 for this component (e.g. setting 1, Table 1). In case of a true device effect, however, the corresponding variable was always selected, including the smaller stopping iterations (e.g. setting 4, Table 1).

To summarize, the results of the simulation suggest that our approach is able to identify device effects on location (systematic bias) and scale (random error), yielding $p$-values that are very similar to the parametric benchmark. For small sample sizes it even showed a slightly better performance with respect to the estimated type I error rate. For high-dimensional data situations the approach works as well as for low-dimensional settings. In this cases, however, it might make sense to use larger values of $m_{\text {stop }}$ in order to give the device variable a fair chance to be selected at all. It is crucial to use the same number of boosting iterations both for the initial model and the permutations, no matter if $m_{\text {stop }}$ was optimized via resampling or not.

\section{Measuring skin pigmentation}

Having fair skin is an important risk factor for melanoma and non-melanoma skin cancer, which is a major public health problem due to increasing incidences. ${ }^{32}$ A common classification scheme in this context is the Fitzpatrick skin-type scale ${ }^{33}$ which is based on a simple questionnaire assessing skin reactivity to ultraviolet (UV) radiation of the unprotected skin. The scale comprises six skin types. Although the Fitzpatrick scale is broadly used in epidemiological studies, over the last years questions arose regarding the practicability of self categorization and the reliability of the scale (see Uter et al. ${ }^{2}$ and references therein). A more objective alternative could be to directly measure constitutive skin pigmentation on skin areas that are typically not exposed to the Sun (e.g. the inner upper arm).

In the epidemiological study that motivated our approach, the skin pigmentation was assessed with two devices: the chromameter CR 300 (Minolta Camera Co., Ltd, Osaka, Japan) and the reflectometer RM 100 (Courage \& Khazaka, Cologne, Germany). 
This design hence represents a special case in the analysis of measurement errors. As no true values are available, we can only compare the measurements from these two devices. From a methodological point of view, this does not change anything regarding the underlying model or the algorithm of our approach. However, the interpretation of the resulting effect estimates changes: in the case of $\hat{\beta}_{\mathrm{sb}} \neq 0$ one could only show that both devices apparently are not measuring the same, and that switching in a study from one device to another may lead to systematic bias. It is, however, impossible to decide which of the devices leads to a smaller systematic bias. In the case of $\hat{\beta}_{\text {re }} \neq 0$, on the other hand, the approach is still able to identify which device has a greater reliability (smaller random error) although no true values are available.

\section{I Data}

The study has been designed as a repeated cross-sectional survey where every summer and winter term all medical students attending the course Biometry and Epidemiology at the Faculty of Medicine of the FAU Erlangen-Nürnberg are invited to participate. ${ }^{34}$

Two devices to measure skin pigmentation were each applied three times by trained members of the study team on the inner upper arm of the students. The chromameter is a tristimulus colour analyser for measuring reflective colours of surfaces. For the purpose of this study, the $Y$ value of the $X Y Z$ CIE 1931 colour system was utilized which can be regarded as a lightness factor. The spectral response curve for the measurement of $Y$ has its peak at approximately $560 \mathrm{~nm}$ with a bandwidth of about $100 \mathrm{~nm}$. The reflectometer is a remission photometry device at $660 \mathrm{~nm}$ wavelength with a bandwidth of $20 \mathrm{~nm}$, scaled from $0 \%$ remission (total black) to $100 \%$ remission (white aluminium oxide, Lambertian surface) at integer values. For further details and discussion see Uter et al. ${ }^{2}$

In total, 2961 measurements from 537 students are available (see Figure 3). Additional covariates include the Fitzpatrick skin type scale, socio-demographic characteristics of the students (e.g. sex, age) as well as categorical variables that can be assumed to be related to skin pigmentation such as



Figure 3. Results from the two devices to measure skin pigmentation. Histograms and corresponding density estimates represent the empirical distribution of the measurements from the chromameter (left) and the reflectometer (right) on the complete study sample. 
hair and iris colour, the usage of sunbeds and the season the measurement was taken (late October to mid November in the winter term and or late April to mid May in the summer term).

\subsection{Results}

First, we fitted a Gaussian location-scale GAMLSS via gamboostLSS with the resulting measurements of each student as outcome variable to select the most influential variables both on the expected value and the variability of the measurement. All available predictors (the indicator variable for the device and also, e.g., the Fitzpatrick skin type) were included via separate linear base-learners both in the candidate models for $\mu$ and for $\sigma$ (the latter via a log-link).

In addition, we included a random effect base-learner, representing a subject-specific intercept, in order to account for the repeated measurements. The optimal number of stopping iterations for the boosting algorithm was selected via 25 -fold bootstrapping on a two-dimensional logarithmic grid.

As could have been already expected from the empirical distribution in Figure 3, the choice of the device had no effect on the expected value of the measurement but on its variability. The baselearner for the device effect was only selected for the additive predictor of $\sigma$, not for $\mu$. Following the model for the $\sigma$ component, the reflectometer has a negative effect on the expected variability compared with the chromameter. As a result, one can conclude that, e.g., changing the device in an epidemiological study from chromameter to reflectometer may not lead to a systematic bias but could have an effect on the random error of the measurement.

As additional predictors we were able to identify the students' hair and iris colour, the Fitzpatrick skin type, the use of sunbeds and the students' weight to be influential for the expected skin pigmentation. For the variability of the measurements, besides the device effect also the iris colour and again the use of sunbeds were selected.

To confirm our results, we re-fitted the same model (including only the variables selected by boosting) with the classical penalized likelihood approach implemented in gamlss. The resulting coefficients for all variables were very similar (results not shown). Due to the random effects, however, the package triggers a warning that the standard errors of the coefficients (and therefore also the $p$-values) could be biased.

To further assess the significance of the device effect on random error, we carried out the proposed permutation approach with $B=1000$. Following the results of the simulation study, we re-fitted the model with a pre-specified fixed $m_{\text {stop }}=1000$ and used the same number also for the permutated samples. As the base-learner for the device indicator was not selected in the initial model for the $\mu$ component one can easily conclude that the null hypothesis (no effect) cannot be rejected: despite the different physical principle for the respective measurements of the two devices outlined above, no significant differences were detected. Thus, both actual devices and their underlying measurement principle, respectively, can be regarded as equivalent concerning the measurement of pigmentation of white human skin. In case of the random error ( $\sigma$ component), none of the 1000 new coefficients from the permutated samples exceeded the initial coefficient $\left(\left|\hat{\beta}_{\text {re }}^{(b)}\right| \geq\left|\hat{\beta}_{\text {re }}\right|\right)$. This leads to the conclusion that the use of the reflectometer yields a highly significant $(p<0.001)$ smaller random error compared with the chromameter, while adjusting for other factors influencing the measurements of skin pigmentation.

\section{Discussion}

In this article, we propose a new approach to analyse measurement errors of medico-technical devices via GAMLSS. The core idea is to fit a GAMLSS for the distribution of the measurements or true values and include the device indicator both in the additive predictors for 
the location and the scale parameter. The advantage of this approach is that it allows to assess potential device effects on the expected value (systematic bias) or on the variance (random error) simultaneously while adjusting for other variables and confounders.

In general, this approach could be followed by all three available inference schemes for GAMLSS: penalized maximum likelihood estimation, ${ }^{20}$ Bayesian inference ${ }^{6}$ and gradient boosting. ${ }^{7}$ We focused on the boosting approach due to its versatile nature and the favourable aspects regarding variable selection and high-dimensional data. However, we confirmed our results from the low-dimensional simulation settings and the application via the penalized likelihood approach, which must be considered as the gold standard for the estimation of GAMLSS.

In our application comparing reflectometer and chromameter for measuring skin pigmentation, we were able to detect a highly significant device effect on the variation of the measurement (random error) while no differences regarding systematic bias were found. As a result, the measurements of both devices can be regarded as equivalent while the larger variability of the chromameter must be considered in practice.

Although our approach was motivated by a very specific application, it also tackles a general methodological problem regarding gradient boosting: due to the implied penalization resulting from stopping the algorithm before it converges (leading to shrinkage of the effect estimates) there is no easy way to generate estimates for the standard errors of the resulting coefficients. It is hence up to now not possible to carry out significant tests and provide $p$-values for models that were fitted by boosting. Via the proposed permutation test we are able to construct realizations of the test statistic under the null hypothesis while taking the special characteristics of the algorithm into account.

This combination of boosting with permutation is in-line with the work of Boulesteix and Hothorn $^{31}$ who proposed a permutation test to assess the additional predictive value of molecular data. The approach is based on permuting the molecular predictors in a logistic regression model (keeping the clinical variables fixed) while using the likelihood ratio as test-statistic.

An advantage of our approach is its flexibility: it can deal with high-dimensional data, can automatically incorporate variable selection for the other predictors and works also when subjectspecific random terms or splines are included in the model. Due to the modular nature of statistical boosting $^{35}$ our test procedure could be easily extended also towards other loss functions (e.g. leading to quantile regression) or distributions in the GAMLSS framework.

A limitation of our approach is that due to its motivation to analyse device effects on measurement errors, it currently assumes the independence of the binary predictor of interest (i.e. the device) to the other covariates. Further research is warranted to possibly extend our concept to significance tests for explanatory variables that are correlated to other predictors. A promising idea in this situation could be to incorporate permutations of regressor residuals. ${ }^{29,30}$

\section{Declaration of conflicting interests}

The author(s) declared no potential conflicts of interest with respect to the research, authorship, and/or publication of this article.

\section{Funding}

The author(s) disclosed receipt of the following financial support for the research, authorship, and/or publication of this article: The work on this article was supported by the Deutsche Forschungsgemeinschaft (http://www.dfg.de, grant number SCHM 2966/1-1) and the Interdisciplinary Center for Clinical Research of the Friedrich-Alexander University Erlangen-Nürnberg (project J49). 


\section{References}

1. Atkinson G and Nevill AM. Statistical methods for assessing measurement error (reliability) in variables relevant to sports medicine. Sports Med 1998; 26(4): 217-238.

2. Uter W, Benz M, Mayr A, et al. Assessing skin pigmentation in epidemiological studies: The reliability of measurements under different conditions. Skin Res Technol 2013; 19(2): 100-106.

3. Rigby RA and Stasinopoulos D. Generalized additive models for location, scale and shape (with discussion). Appl Statist 2005; 54: 507-554.

4. Hastie T and Tibshirani R. Generalized Additive Models. London: Chapman \& Hall, 1990.

5. Rigby RA and Stasinopoulos DM. Automatic smoothing parameter selection in GAMLSS with an application to centile estimation. Statist Meth Med Res 2014; 23(4): 346-368.

6. Klein N, Kneib T and Lang S. Bayesian generalised additive models for location, scale, and shape for zeroinflated and overdispersed count data. Journal of the American Statistical Association (JASA) 2015. DOI:10.1080/01621459.2014.912955.

7. Mayr A, Fenske N, Hofner B, et al. Generalized additive models for location, scale and shape for high-dimensional data - a flexible approach based on boosting. $J R$ Statist Soc Ser C Appl Statist 2012; 61(3): 403-427.

8. Kneib T. Beyond mean regression. Statist Modell 2013; 13(4): 275-303.

9. Fenske N, Kneib T and Hothorn T. Identifying risk factors for severe childhood malnutrition by boosting additive quantile regression. JASA 2011; 106(494): 494-510.

10. Hothorn T, Kneib T and Bühlmann P. Conditional transformation models. $J$ R Statist Soc Ser B Statist Methodol 2014; 76(1): 3-27.

11. van Buuren S. Growth charts of human development. Statist Meth Med Res 2014; 23(4): 346-368.

12. van Dommelen $P$ and van Buuren S. Methods to obtain referral criteria in growth monitoring. Statist Meth Med Res 2014; 23(4): 369-389.

13. De Onis M and WHO Multicentre Growth Reference Study Group. WHO child growth standards: length/ height-for-age, weight-for-age, weight-for-length, weightfor-height and body mass index-for-age: Methods and development. Geneva: World Health Organization 2006; Available at: http://www.who.int/iris/handle/10665/43413.

14. Wahl S, Fenske N, Zeilinger S, et al. On the potential of models for location and scale for genome-wide DNA methylation data. BMC Bioinform 2014; 15(1): 232.

15. Muniz-Terrera G, van den Hout A, Rigby $R$ et al. Analysing cognitive test data: Distributions and nonparametric random effects. Statist Meth Med Res 2016; 25 : $741-753$.

16. Bühlmann $\mathrm{P}$ and Hothorn T. Boosting algorithms: Regularization, prediction and model fitting (with discussion). Statist Sci 2007; 22: 477-522.

17. Hofner B, Mayr A, Robinzonov N, et al. Model-based boosting in R: A hands-on tutorial using the $\mathrm{R}$ package mboost. Computat Statist 2014; 29(1-2): 3-35.
18. Schmid M, Wickler F, Maloney KO, et al. Boosted beta regression. PLoS ONE 2013; 8(4): e61623.

19. Schmid M, Potapov S, Pfahlberg A, et al. Estimation and regularization techniques for regression models with multidimensional prediction functions. Statist Comput 2010; 20: 139-150.

20. Stasinopoulos D and Rigby R. Generalized additive models for location scale and shape (GAMLSS) in R. J Statist Softw 2007; 23(7): 1-46.

21. Mayr A, Binder H, Gefeller O, et al. The evolution of boosting algorithms - from machine learning to statistical modelling. Meth Inform Med 2014; 53(6): 419-427.

22. Mayr A, Binder H, Gefeller O, et al. Extending statistical boosting - an overview of recent methodological developments. Meth Inform Med 2014; 53(6): 428-435.

23. Hastie T, Tibshirani R and Friedman J. The Elements of Statistical Learning: Data Mining, Inference, and Prediction, 2nd edn. New York: Springer, 2009.

24. Mayr A, Hofner B and Schmid M. The importance of knowing when to stop - a sequential stopping rule for component-wise gradient boosting. Meth Inform Med 2012; 51(2): 178-186.

25. R Core Team. R: A Language and Environment for Statistical Computing. R Foundation for Statistical Computing, Vienna, Austria, 2014. http://www.R-project.org/

26. Hofner B, Mayr A and Schmid M. gamboostlss: An R package for model building and variable selection in the gamlss framework. Preprint arXiv:14071774, 2014.

27. Draper NR and Stoneman DM. Testing for the inclusion of variables in linear regression by a randomisation technique. Technometrics 1966; 8(4): 695-699.

28. Kennedy PE and Cade BS. Randomization tests for multiple regression. Commun Statist Sim Computat 1996; 25(4): 923-936.

29. Potter DM. A permutation test for inference in logistic regression with small and moderate-sized data sets. Statist Med 2005; 24(5): 693-708.

30. Werft W and Benner A. glperm: A permutation of regressor residuals test for inference in generalized linear models. The R Journal 2010; 2(1): 39-43.

31. Boulesteix AL and Hothorn T. Testing the additional predictive value of high-dimensional molecular data. $B M C$ Bioinformatics 2010; 11: 78DOI:10.1186/1471-2105-11-78.

32. Borges P, Rodrigues J, Louzada F et al. A cure rate survival model under a hybrid latent activation scheme. Statist Meth Med Res 2016; 25: 838-856.

33. Fitzpatrick TB. The validity and practicality of Sunreactive skin types I through VI. Arch Dermatol 1988; 124(6): 869-871.

34. Fiessler C, Pfahlberg A, Li J, et al. Accuracy and reliability of naevus self-counts. Melanoma Res 2014; 24(6): 611-616.

35. Bühlmann P, Gertheiss J, Hieke S, et al. Discussion of "The evolution of boosting algorithms" and "Extending statistical boosting". Meth Inf Med 2014; 53: $436-445$.

\section{Appendix}

\section{The gamboostLSS algorithm for boosting GAMLSS}

Let $\boldsymbol{\theta}=\left(\theta_{k}\right)_{k=1, . ., K}$ be the vector of distribution parameters (e.g. $(\mu, \sigma, v, \tau)$ ) of a GAMLSS. Each distribution parameter has its own additive predictor $\eta_{\theta_{k}}$ and associated link function $g_{k}$ so that 
$\theta_{k}=g_{k}^{-1}\left(\eta_{\theta_{k}}\right)$. The gamboostLSS algorithm ${ }^{7}$ circles between the different distribution parameters $\theta_{k}, k=1, \ldots, K$, and fits all base-learners $h(\cdot)$ separately to the negative partial derivatives of the loss function, i.e. to the partial derivatives of the log-likelihood with respect to the additive predictors $\eta_{\theta_{k}}$, i.e. $\frac{\partial}{\partial \eta_{\theta_{k}}} l(\boldsymbol{y}, \boldsymbol{\theta})$.

\section{Initialize}

(1) Initialize the additive predictors $\hat{\eta}_{\mu_{i}}^{[0]}, \hat{\eta}_{\sigma_{i}}^{[0]}, \hat{\eta}_{\nu_{i}}^{[0]}, \hat{\eta}_{\tau_{i}}^{[0]}$ with offset values, e.g. $\hat{\eta}_{\theta_{k i}}^{[0]}=\underset{c}{\arg \max } \sum_{i=1}^{n} l\left(y_{i}, \eta_{\theta_{k i}}=c\right)$ for $k=1, \ldots, 4$ and $i=1, \ldots, n$. Set the iteration counter $m=0$.

(2) For each distribution parameter $\theta_{k}, k=1, \ldots, 4$, specify a set of base-learners: i.e. for parameter $\theta_{k}$ by $h_{k 1}(\cdot), \ldots, h_{k p_{k}}(\cdot), k=1, \ldots, 4$, where $p_{k}$ is the cardinality of the set of base-learners specified for $\theta_{k}$.

\section{Boosting in multiple dimensions}

(3) Start a new boosting iteration: increase $m$ by 1 and set $k:=0$.

(4) Increase $k$ by 1.

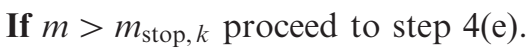

Otherwise compute the partial derivative $\frac{\partial}{\partial \eta_{\theta_{k}}} l\left(\boldsymbol{y}, \boldsymbol{\theta}_{i}\right)$ by plugging in the current estimates $\boldsymbol{\eta}_{i}=\left(\hat{\eta}_{\mu_{i}}^{[m-1]}, \hat{\eta}_{\sigma_{i}}^{[m-1]}, \hat{\eta}_{v_{i}}^{[m-1]}, \hat{\eta}_{\tau_{i}}^{[m-1]}\right)$ :

$$
\boldsymbol{u}_{k}^{[m-1]}=\left(\frac{\partial}{\partial \eta_{\theta_{k}}} l\left(y_{i}, \boldsymbol{\eta}_{i}\right)\right)_{i=1, \ldots, n}
$$

(b) Fit the gradient vector $\boldsymbol{u}_{k}^{[m-1]}$ to each of the base-learners contained in the set of base-learners specified for the predictor $\eta_{\theta_{k}}$ in step (2).

(c) Select the component $j^{*}$ that best fits the gradient vector according to the least-squares criterion, i.e. select the base-learner $h_{k j^{*}}$ defined by

$$
j^{*}=\underset{1 \leq \delta \leq p_{k}}{\arg \min } \sum_{i=1}^{n}\left(u_{i k}^{[m-1]}-h_{k j}(\cdot)\right)^{2} .
$$

(d) Update the additive predictor $\eta_{\theta_{k}}$ as follows:

$$
\hat{\eta}_{\theta_{k}}^{[m-1]}:=\hat{\eta}_{\theta_{k}}^{[m-1]}+\mathrm{sl} \cdot h_{k j^{*}}(\cdot)
$$

where sl is a small step-length $(0<\mathrm{sl} \ll 1)$.

(e) Set $\hat{\eta}_{\theta_{k}}^{[m]}:=\hat{\eta}_{\theta_{k}}^{[m-1]}$.

(f) Iterate steps 4(a)-(e) for $k=2, \ldots, 4$.

\section{Iterate}


(5) Iterate steps 3 and 4 until $m>m_{\text {stop, } k}$ for all $k=1, \ldots, 4$.

\section{Example R-code}

The gamboostLSS algorithm can be easily applied in practice via the $\mathrm{R}$ add-on package gamboostLSS. A detailed tutorial containing a worked-out case study can be found in Hofner et al. ${ }^{26}$

The implementation of the additional permutation test will be demonstrated in a small simulated example:

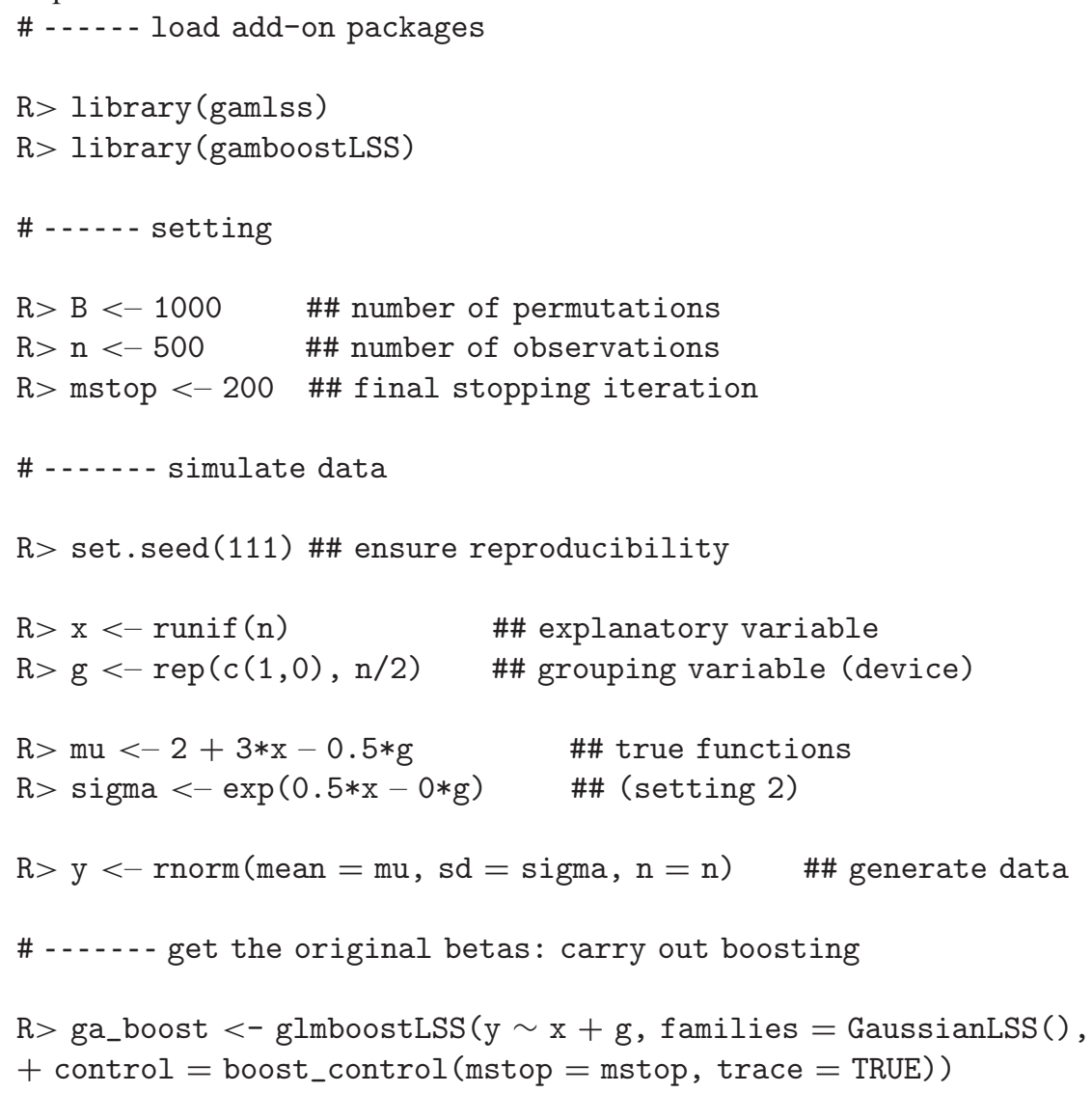






\#\# get the parametric p-values from gamlss

$\mathrm{R}>$ ga_orig <- gamlss $(\mathrm{y} \sim \mathrm{x}+\mathrm{g}$, sigma.formula $=\sim \mathrm{x}+\mathrm{g})$

GAMLSS-RS iteration 1: Global Deviance $=1653.05$

GAMLSS-RS iteration 2: Global Deviance $=1653.04$

GAMLSS-RS iteration 3: Global Deviance $=1653.04$

$\mathrm{R}>$ p.gamlss.mu <- round (summary (ga_orig) $[3,4], 3$ )

$\mathrm{R}>$ p.gamlss.si <- round (summary (ga_orig) $[6,4], 3$ )

$\mathrm{R}>$ cbind (boosting $=\mathrm{c}(\mathrm{mu}=$ p.boost.mu, sigma $=$ p.boost.si),

+ parametric $=c(m u=p \cdot g a m l s s . m u$, sigma $=$ p.gamlss.si $))$

boosting parametric

$\begin{array}{lll}\mathrm{mu} & 0.000 \quad 0.000\end{array}$

$\begin{array}{lll}\text { sigma } & 0.813 \quad 0.822\end{array}$ 